\title{
Crystal structure of diaquasuberatocobalt(II), $\mathrm{Co}\left(\mathrm{C}_{8} \mathrm{H}_{12} \mathrm{O}_{4}\right)\left(\mathrm{H}_{2} \mathrm{O}\right)_{2}$
}

\author{
Y.-Q. Zheng*, J.-L. Lin, J. Sun and A.-Y. Pan
}

Ningbo University, Institute for Solid State Chemistry, Ningbo, Zhejiang, 315211 P. R. China

Received August 2, 1999, CCDC-No. 1267/256

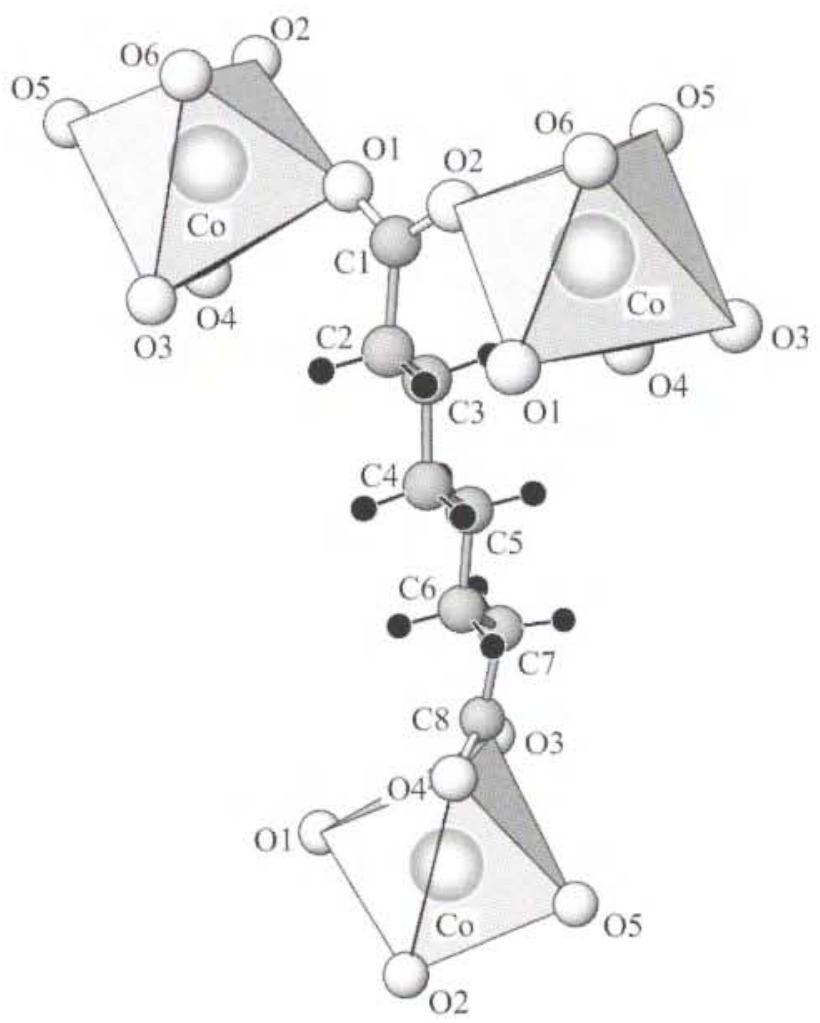

Co atom and the other bridge two Co atoms terminally. The Co atom is octahedrally coordinated by six oxygen atoms $\left(2 \mathrm{H}_{2} \mathrm{O}\right.$, 1 carboxyl chelate, 2 terminal bridging carboxyl with $d(\mathrm{Co}-\mathrm{O})$ $=2.043 \AA-2.158 \AA$. The $\mathrm{C}$ atoms and the chelating $\mathrm{O}$ atoms of the $\alpha, \omega-\mathrm{OOC}\left(\mathrm{CH}_{2}\right)_{6} \mathrm{COO}$ dicarboxylate anion are nearly coplanar with $d(\mathrm{C}-\mathrm{C})=1.504 \overline{\mathrm{A}}-1.526 \AA$ and $<(\mathrm{C}-\mathrm{C}-\mathrm{C})=$ $111.5^{\circ}-114.1^{\circ}$. The second carboxyl group is twisted by about $90 \%\left(d(\mathrm{C}-\mathrm{O})=1.257 \AA-1.258 \AA\right.$ and $\varangle(\mathrm{O}-\mathrm{C}-\mathrm{O})=118.7(3)^{\circ}$, $\left.120.5(3)^{\circ}\right)$. One of the $\mathrm{H}_{2} \mathrm{O}$ molecules $(d(\mathrm{O}-\mathrm{H})=0.75 \AA$ $\left.-0.84 \AA, \varangle(\mathrm{H}-\mathrm{O}-\mathrm{H})=109^{\circ}\right)$ forms hydrogen bonds to two carboxyl $\mathrm{O}$ atoms with $d(\mathrm{O} \cdots \mathrm{O})=2.721 \AA, 2.759 \AA$ and $\measuredangle(\mathrm{O}-\mathrm{H} \cdots \mathrm{O})$ $=158.3^{\circ}, 173.1^{\circ}$, and the other to one carboxyl $\mathrm{O}$ atom and another crystallographically different $\mathrm{H}_{2} \mathrm{O}$ molecule with $d(\mathrm{O} \cdots \mathrm{O})$ $=2.811 \AA, 2.863 \AA$ and $\varangle(\mathrm{O}-\mathrm{H} \cdots \mathrm{O})=171.8^{\circ}, 162.1^{\circ}$.

Table 1. Data collection and handling.

Crystal:

Wavelength:

$\mu$ :

Diffractometer, scan mode: $2 \theta_{\max }$ :

$N(h k l)_{\text {measured, }} N(h k l)_{\text {unique }}$

Criterion for $I_{\mathrm{obs}}, N(h k l)_{\mathrm{gt}}$ N(param $)_{\text {refined }}$

Programs: rose-colored block, size $0.08 \times 0.12 \times 0.15 \mathrm{~mm}$ Mo $K_{\alpha}$ radiation $(0.71073 \AA)$ $16.33 \mathrm{~cm}^{-1}$

Bruker $\mathrm{P} 4, \boldsymbol{\theta} / 2 \theta$ $54.98^{\circ}$

3278,2425

$I_{\text {obs }}>2 \sigma\left(I_{\text {obs }}\right), 1582$ 142

SHELXS-97 [1], SHELXL-97 [2]

\begin{abstract}
$\mathrm{C}_{8} \mathrm{H}_{16} \mathrm{CoO}_{6}$, monoclinic, $P 12{ }_{1} / c 1$ (No. 14), $a=14.856(2) \AA$, $b=7.449(1) \AA, c=9.614(1) \AA, \beta=97.44(1)^{\circ}, V=1055.0 \AA^{3}$, $Z=4, R_{\mathrm{gt}}(F)=0.043, w R\left(F^{2}\right)=0.096, T=293 \mathrm{~K}$.
\end{abstract}

\section{Source of material}

The title compound was synthesized by the reaction of $0.5 \mathrm{~g}$ $\left(2.90 \mathrm{mmol}\right.$ ) suberic acid with an excess of fresh $\mathrm{CoCO}_{3}$ in $25 \mathrm{ml}$ twice-destilled water, followed by filtrating the unreacted $\mathrm{CoCO}_{3}$. The filtrate was then maintained at $331 \mathrm{~K}$ and rose-colored crystals formed within two days.

\section{Discussion}

The crystal structure consists of ${ }_{\infty}^{2}\left[\mathrm{Co}\left(\mathrm{H}_{2} \mathrm{O}\right)_{2}\left(\mathrm{C}_{8} \mathrm{H}_{12} \mathrm{O}_{4}\right)_{3 / 3}\right]$ layers with $\mathrm{H}_{2} \mathrm{O}$ molecules between. $\alpha, \omega-\mathrm{OOC}\left(\mathrm{CH}_{2}\right)_{6} \mathrm{COO}$ zig-zag chains parallel to [100] are fragments of the 2D layers which extend parallel to (100) and are held together by hydrogen bonds. Each $\alpha, \omega-\mathrm{OOC}\left(\mathrm{CH}_{2}\right)_{6} \mathrm{COO}$ dicarboxylate anion links three $\mathrm{Co}$ atoms. One terminal carboxyl group acts as chelat ligand to one
Table 2. Atomic coordinates and displacement parameters (in $\dot{A}^{2}$ ).

\begin{tabular}{llllll}
\hline Atom & Site & $x$ & $y$ & $z$ & $U_{\text {iso }}$ \\
\hline $\mathrm{Ha}(\mathrm{O} 5)$ & $4 e$ & 0.5296 & 0.2041 & 0.5340 & $0.05(1)$ \\
$\mathrm{Hb}(\mathrm{O})$ & $4 e$ & 0.6191 & 0.2284 & 0.5260 & $0.10(2)$ \\
$\mathrm{Ha}(\mathrm{O} 6)$ & $4 e$ & 0.4472 & 0.0895 & 0.7282 & $0.06(2)$ \\
$\mathrm{Hb}(\mathrm{O} 6)$ & $4 e$ & 0.4575 & -0.0573 & 0.7912 & $0.06(2)$ \\
$\mathrm{H}(2 \mathrm{~A})$ & $4 e$ & 0.7296 & -0.4674 & 0.5696 & $0.047(3)$ \\
$\mathrm{H}(2 \mathrm{~B})$ & $4 e$ & 0.7296 & -0.3109 & 0.6788 & $0.047(3)$ \\
$\mathrm{H}(3 \mathrm{~A})$ & $4 e$ & 0.8186 & -0.2974 & 0.4354 & $0.047(3)$ \\
$\mathrm{H}(3 \mathrm{~B})$ & $4 e$ & 0.8137 & -0.1318 & 0.5348 & $0.047(3)$ \\
$\mathrm{H}(4 \mathrm{~A})$ & $4 e$ & 0.8976 & -0.2981 & 0.7196 & $0.047(3)$ \\
$\mathrm{H}(4 \mathrm{~B})$ & $4 e$ & 0.9066 & -0.4552 & 0.6139 & $0.047(3)$ \\
$\mathrm{H}(5 \mathrm{~A})$ & $4 e$ & 0.9892 & -0.2577 & 0.4818 & $0.047(3)$ \\
$\mathrm{H}(5 \mathrm{~B})$ & $4 e$ & 0.9863 & -0.1127 & 0.5991 & $0.047(3)$ \\
$\mathrm{H}(6 \mathrm{~A})$ & $4 e$ & 1.0765 & -0.4447 & 0.6432 & $0.047(3)$ \\
$\mathrm{H}(6 \mathrm{~B})$ & $4 e$ & 1.0743 & -0.2981 & 0.7594 & $0.047(3)$ \\
$\mathrm{H}(7 \mathrm{~A})$ & $4 e$ & 1.1570 & -0.2356 & 0.5157 & $0.047(3)$ \\
$\mathrm{H}(7 \mathrm{~B})$ & $4 e$ & 1.1631 & -0.1074 & $0.645 \overline{7}$ & $0.047(3)$ \\
\hline
\end{tabular}

\footnotetext{
* Correspondence author (e-mail: zhengcm@nbu.edu.cn)
} 
Table 3. Atomic coordinates and displacement parameters (in $\AA^{2}$ ).

\begin{tabular}{|c|c|c|c|c|c|c|c|c|c|c|}
\hline Atom & Site & $x$ & $y$ & $z$ & $U_{11}$ & $U_{22}$ & $U_{33}$ & $U_{12}$ & $U_{13}$ & $U_{23}$ \\
\hline Co & $4 e$ & $0.61398(3)$ & $0.01039(7)$ & $0.72373(4)$ & $0.0309(2)$ & $0.0407(3)$ & $0.0274(2)$ & $-0.0010(2)$ & $0.0045(2)$ & $-0.0015(2)$ \\
\hline$O(1)$ & $4 e$ & $0.6284(2)$ & $-0.2934(3)$ & $0.3671(2)$ & $0.041(1)$ & $0.047(1)$ & $0.026(1)$ & $0.003(1)$ & $0.001(1)$ & $-0.006(1)$ \\
\hline$O(2)$ & $4 e$ & $0.5937(2)$ & $-0.1607(4)$ & $0.5560(2)$ & $0.034(1)$ & $0.050(2)$ & $0.032(1)$ & $0.004(1)$ & $0.002(1)$ & $-0.009(1)$ \\
\hline$O(3)$ & $4 e$ & $1.3206(2)$ & $-0.2939(4)$ & $0.6315(3)$ & $0.036(1)$ & $0.058(2)$ & $0.038(1)$ & $0.003(1)$ & $0.009(1)$ & $0.009(1)$ \\
\hline$O(4)$ & $4 e$ & $1.2463(2)$ & $-0.4339(4)$ & $0.7788(3)$ & $0.034(1)$ & $0.059(2)$ & $0.045(2)$ & $0.002(1)$ & $0.007(1)$ & $0.016(1)$ \\
\hline $\mathrm{O}(5)$ & $4 e$ & $0.5784(2)$ & $0.2269(3)$ & $0.5795(3)$ & $0.035(1)$ & $0.046(2)$ & $0.033(1)$ & $-0.002(1)$ & $0.003(1)$ & $-0.001(1)$ \\
\hline$O(6)$ & $4 e$ & $0.4823(2)$ & $0.0199(4)$ & $0.7637(3)$ & $0.035(1)$ & $0.052(2)$ & $0.060(2)$ & $0.005(1)$ & $0.012(1)$ & $0.016(2)$ \\
\hline$C(1)$ & $4 e$ & $0.6454(2)$ & $-0.2603(5)$ & $0.4966(3)$ & $0.034(2)$ & $0.034(2)$ & $0.032(2)$ & $-0.007(1)$ & $0.003(1)$ & $-0.000(2)$ \\
\hline $\mathrm{C}(2)$ & $4 e$ & $0.7299(2)$ & $-0.3379(5)$ & $0.5802(4)$ & $0.034(2)$ & $0.047(2)$ & $0.032(2)$ & $0.004(2)$ & $0.004(1)$ & $-0.002(2)$ \\
\hline$C(3)$ & $4 e$ & $0.8161(2)$ & $-0.2619(5)$ & $0.5319(4)$ & $0.034(2)$ & $0.048(2)$ & $0.038(2)$ & $0.004(2)$ & $0.005(2)$ & $0.001(2)$ \\
\hline$C(4)$ & $4 e$ & $0.9021(2)$ & $-0.3259(6)$ & $0.6222(4)$ & $0.034(2)$ & $0.057(2)$ & $0.040(2)$ & $0.004(2)$ & $0.004(2)$ & $0.002(2)$ \\
\hline $\mathrm{C}(5)$ & $4 e$ & $0.9882(2)$ & $-0.2409(6)$ & $0.5816(4)$ & $0.038(2)$ & $0.055(2)$ & $0.043(2)$ & $0.004(2)$ & $0.004(2)$ & $0.007(2)$ \\
\hline$C(6)$ & $4 e$ & $1.0749(2)$ & $-0.3164(6)$ & $0.6597(4)$ & $0.035(2)$ & $0.053(2)$ & $0.043(2)$ & $0.002(2)$ & $0.005(2)$ & $0.005(2)$ \\
\hline$C(7)$ & $4 e$ & $1.1605(2)$ & $-0.2324(5)$ & $0.6171(4)$ & $0.035(2)$ & $0.044(2)$ & $0.048(2)$ & $0.004(2)$ & $0.007(2)$ & $0.004(2)$ \\
\hline $\mathrm{C}(8)$ & $4 e$ & $1.2465(2)$ & $-0.3240(5)$ & $0.6795(4)$ & $0.036(2)$ & $0.040(2)$ & $0.033(2)$ & $0.000(2)$ & $0.004(1)$ & $-0.003(2)$ \\
\hline
\end{tabular}

Acknowledgments. The project was sponsered by the Scientific Research Foundation for the returned Overseas Chinese Scholars, State Educational Ministry and the Bruker P4 diffractometer was purchased by the generous support of Ningbo Scientific-Technical Commision and Ningbo Eucational Committee.

\section{References}

1. Sheldrick, G. M.: Phase Annealing in SHELX-90: Direct Methods for Larger Structures. Acta Crystallogr. A46 (1990) 467-473.

2. Sheldrick, G. M.: SHELXL-97. Program for the Refinement of Crystal Structures. University of Göttingen, Germany 1997. 